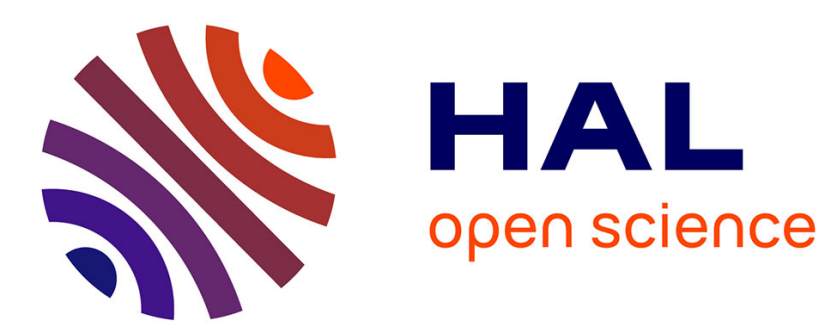

\title{
A novel coupled transmission-reflection tomography and the V-line Radon transform
}

\author{
Rémi Regnier, M.K. Nguyen
}

\section{To cite this version:}

Rémi Regnier, M.K. Nguyen. A novel coupled transmission-reflection tomography and the V-line Radon transform. 2011 18th IEEE International Conference on Image Processing, Sep 2011, Bruxelles, Belgium. hal-00797454

\section{HAL Id: hal-00797454 https://hal.science/hal-00797454}

Submitted on 6 Mar 2013

HAL is a multi-disciplinary open access archive for the deposit and dissemination of scientific research documents, whether they are published or not. The documents may come from teaching and research institutions in France or abroad, or from public or private research centers.
L'archive ouverte pluridisciplinaire HAL, est destinée au dépôt et à la diffusion de documents scientifiques de niveau recherche, publiés ou non, émanant des établissements d'enseignement et de recherche français ou étrangers, des laboratoires publics ou privés. 


\title{
A novel coupled transmission-reflection tomography and the V-line Radon transform
}

\author{
Rémi Régnier, and Mai K. Nguyen,
}

\begin{abstract}
The Radon transform $(R T)$ on straight lines deals as mathematical foundation for conventional tomographic imaging (e.g. X-ray scanner, Single Photon Emission Computed Tomography (SPECT), Positron Emission Tomography (PET)) which use only one physical phenomenon, i.e. either transmission or emission of radiation. An imaging concept exploiting jointly two phenomena leads to a Radon transform defined on a geometrical support different from straight lines. In this paper, we propose a new two-dimensional $X$-ray imaging based on the coupling between transmission and reflection. Its modeling leads to a Radon transform defined on a pair of half-lines forming a vertical letter $\mathbf{V}$, called $\mathbf{V}$-line $R T(V-R T)$. Moreover we establish the analytic inverse formula of this new transform, which forms the mathematical basis for image reconstruction. Through simulations, image formation and reconstruction results show the feasibility of this new imaging. The main advantage is the use of an one-dimensional detector which does not rotate for two-dimensional image reconstruction.
\end{abstract}

Index Terms-X-ray imaging, tomography, reflection, mirrors, Radon transform.

\section{INTRODUCTION}

While the classical Radon transform defined on straight rays models the imaging systems (X-ray scanner, SPECT) which need a relative rotation between the object and the detector ([1], [2], [3]), there exists a considerable interest for systems which do not require such a relative rotation and a two-dimensional detector for two-dimensional image reconstruction. The modeling of the last systems leads to the $R T$ defined on other geometries than the straight-lines.

In this paper, we propose a new tomographic modality which operate with X-ray. But instead of simply analyzing the absorption coefficients on a straight line as in X-ray scanner, we place a special mirror capable of reflecting radiation and so we project the absorption function on a pair of half-lines (and no longer on a straight line). This variant of the tomography would allow to replace the rotation of source-detector couple by setting a series of point-sources and a series of point-detectors. So using a one dimensional non rotating detector for two-dimensional image reconstruction turns out to be an attractive alternative to conventional tomography.

Mathematically, the modeling of this coupled transmissionreflection tomography leads to a Radon transform defined

Rémi Régnier and Mai K. Nguyen are with the Laboratory Equipe Traitement de l'Information et Systèmes, CNRS UMR 8051/ENSEA/Université de Cergy-Pontoise, mail: (see regnierremi@yahoo.fr, mai.nguyen-verger@ucergy.fr). on a pair of half-lines forming a V-letter, called V-line $R T$ $(V-R T)$. This transform is a member of the family of the so-called $T V$-transform ([4], [5], [6], [7], [8]). The simple $T V$-transform was first proposed by Basko [9] in 1997 as a model for image formation in a two-dimensional Compton camera. However this Basko transform is in fact a V-line Radon transform with swinging axis around a detection site whereas the one considered here has a fixed axis direction [4]. The V-line Radon transform is also considered as a two-dimensional version of the Conical Radon Transform $(C R T)$ which have been introduced some years ago ([5], [10], [11], [12], [13]). These generalized Radon transforms ( $T V$ and $C R T$ ) have numerous applications in imaging science, in particular in new Compton scattering emission imaging ([4], [5], [6], [7], [8], [10], [11], [12], [13]).

In this paper a new application of the $T V$-transform is developed in primary radiation transmission imaging, more precicely as a coupled transmission-reflection tomography.

In this new tomography, X-radiation emitted from the source falls on the mirror under an incidence angle and registered later by a detector (see Fig.1). In this way when the object is scanned by all possible V-lines, then the standard rotational motion can be avoided.

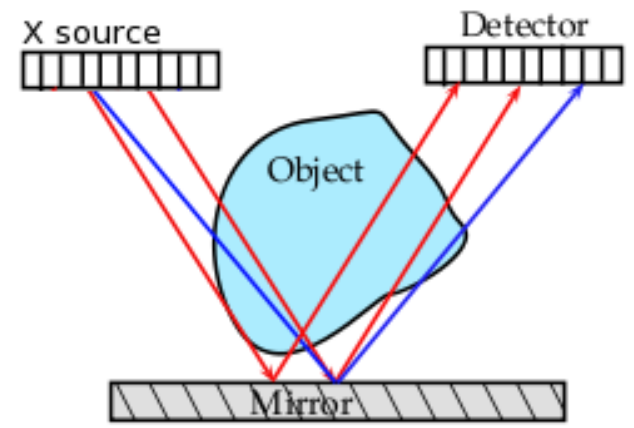

Fig. 1. The X-ray source can be translated (see red rays) and emits according to some angle (see the blue rays) which generates enough data on the detector to reconstruct the attenuation map.

In section 2 we present the modeling of the new transmission-reflection imaging concept which leads to the V-line Radon transform. Then we establish its analytic inverse and derive a corresponding filtered back-projection form. This last form has the advantage of reconstructing the image by fast algorithms.

In section 3, we present numerical simulation results on 
image formation and reconstruction to support the feasibility of this new imaging. The paper ends with a short conclusion on the obtained results and opens some future research perspectives.

\section{MODELING OF THE TRANSMISSION-REFLEXION IMAGING AND THE V-LINE RADON TRANSFORM}

\section{A. Image formation modeling and the V-line Radon transform} $(V-R T)$

In tomographic transmission imaging, the attenuation map of a two-dimensional object is represented by a non negative continuous function $f(x, y)$ with bounded support. A linear detector collects the transmitted radiation after a reflection on a mirror. We may assume that the reflection coefficient of the mirror is of the form $e^{-c(\theta)}$, where $\theta$ is the angle of incidence. From now on, we consider the ideal case where $c(\theta)=0$. This does not affect the validity of the modeling.

Each measurement on the detector represents the sum of the radiation attenuation along a trajectory in the shape of letter $\mathrm{V}$, since photons travel from the point of emission at the source up to the point of detection after a reflection on the mirror. Thus $f(x, y)$ is integrated on a discontinuous line having the form of the V-line with a symmetry axis parallel to the $O y$ direction (see Fig.2).

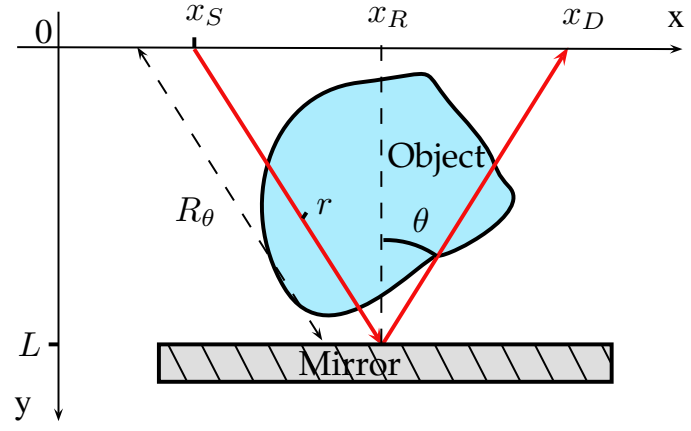

Fig. 2. Parameters of the V-line Radon transform

The projection data $\widehat{g}\left(x_{R}, \theta\right)$ on the detector can be written as

$$
\begin{aligned}
\widehat{g}\left(x_{R}, \theta\right) & =\int_{0}^{\infty} f\left(x_{R}+r \sin (\theta), r \cos (\theta)\right) d r \\
& +\int_{0}^{\infty} f\left(x_{R}-r \sin (\theta), r \cos (\theta)\right) d r .
\end{aligned}
$$

Equation (1) gives $\widehat{g}\left(x_{R}, \theta\right)$, as the V-line Radon transform of the unknown attenuation distribution $f(x, y)$. This is the image formation equation in the transmission-reflection imaging procedure.

\section{$B$. The inverse transformation $(V-R T)^{-1}$ and image recon- struction}

For convenience of notations, let us define $g\left(x_{R}, t\right)=$ $\widehat{g}\left(x_{R}, \theta\right)$, with $t=\tan \theta$. The inverse transform $(V-R T)^{-1}$ can be worked out using Fourier transforms $\tilde{f}(q, y)$ (resp. $\tilde{g}(q, t))$ with respect to the variable $x$ (resp. $\left.\left(x_{R}\right)\right)$ of $f(x, y)$ (resp. $g\left(x_{R}, t\right)$ ),

$$
\begin{aligned}
g\left(x_{R}, t\right) & =\int_{-\infty}^{\infty} \tilde{g}(q, t) e^{2 i \pi q x_{R}} d q \\
f(x, y) & =\int_{-\infty}^{\infty} \tilde{f}(q, y) e^{2 i \pi q x} d q .
\end{aligned}
$$

Equation (1) becomes now with the change of variable $z=$ $r \cos (\theta)$

$$
\begin{gathered}
\frac{\tilde{g}(q, t)}{\sqrt{1+t^{2}}}=\int_{0}^{\infty} \tilde{f}(q, z) 2 \cos (2 \pi q z t) d z \text { for } \theta \in[0, \pi / 2] \\
\frac{\tilde{g}(q,-t)}{\sqrt{1+t^{2}}}=\int_{0}^{\infty} \tilde{f}(q,-z) 2 \cos (2 \pi q z t) d z \text { for } \theta \in[-\pi / 2,0] .
\end{gathered}
$$

We multiply equations (4) on both sides by $\int_{0}^{\infty} 2 \cos (2 \pi z t) d(q t)$ and using the identity

$$
\int_{0}^{\infty} 4 \cos (2 \pi \eta x) \cos \left(2 \pi \eta^{\prime} x\right) d x=\delta\left(\eta+\eta^{\prime}\right)+\delta\left(\eta-\eta^{\prime}\right),
$$

we obtain

$$
\begin{aligned}
\tilde{f}(q, z) & =|q| \int_{0}^{\infty} \frac{\tilde{g}(q, t)}{\sqrt{1+t^{2}}} 2 \cos (2 \pi q t z) d t, \\
\tilde{f}(q,-z) & =|q| \int_{0}^{\infty} \frac{\tilde{g}(q,-t)}{\sqrt{1+t^{2}}} 2 \cos (2 \pi q t z) d t .
\end{aligned}
$$

So for the full angular range, we can write

$$
\begin{array}{r}
\tilde{f}(q, z)=|q| \int_{0}^{\infty} 2 \cos (2 \pi q t z) \cdot U(z) \tilde{g}(q, t) \frac{d t}{\sqrt{1+t^{2}}} \\
\quad+|q| \int_{0}^{\infty} 2 \cos (2 \pi q t z) \cdot U(-z) \tilde{g}(q,-t) \frac{d t}{\sqrt{1+t^{2}}},
\end{array}
$$

where $\mathrm{U}$ is the Heaviside unit step function. By inverse Fourier transform we recover

$$
\begin{array}{r}
f(x, z)=\int_{-\infty}^{\infty} d q e^{2 \pi i q x}|q| \\
{\left[\int_{0}^{\infty} 2 \cos (2 \pi q t z) \cdot U(z) \tilde{g}(q, t) \frac{d t}{\sqrt{1+t^{2}}}\right.} \\
\left.+\int_{0}^{\infty} 2 \cos (2 \pi q t z) \cdot U(-z) \tilde{g}(q,-t) \frac{d t}{\sqrt{1+t^{2}}}\right] .
\end{array}
$$

This formula is also a so-called filtered back-projection method for image reconstruction [5]. It has the advantage of offering fast algorithms. Technically the back-projection operation consists in assigning the value $g\left(x_{R}, t\right)=\widehat{g}\left(x_{R}, \theta\right)$ to every point on the "projection" V-line, which has given rise to this value, and then to sum over all contributions for every V-line "projections". Therefore we shall follow this procedure in our numerical simulations in the next section.

\section{NUMERICAL SIMULATIONS}

In this section, we simulate image formation and reconstruction using the $V-R T$ and its inverse for a point object and for a Shepp-Logan phantom (Fig. 3,6)). The original images are of size $128 \times 128$ of length units and the angular sampling rate is $d \theta=0.005$ rad. Fig. 4 shows the $V-R T$ transform of the point object. It is also called the point spread function (PSF) of the transform. 


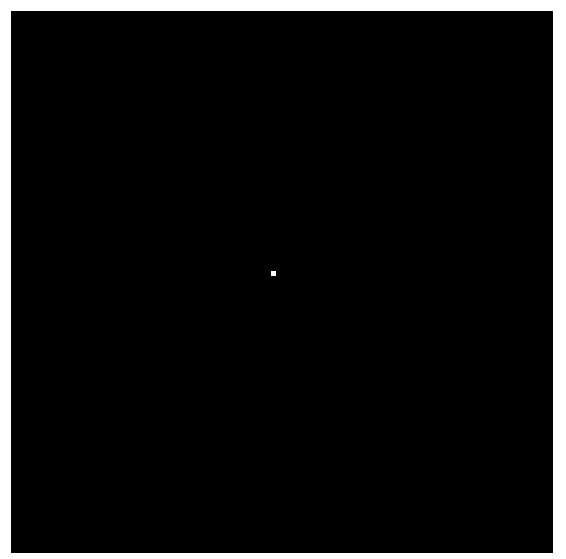

Fig. 3. Original point object

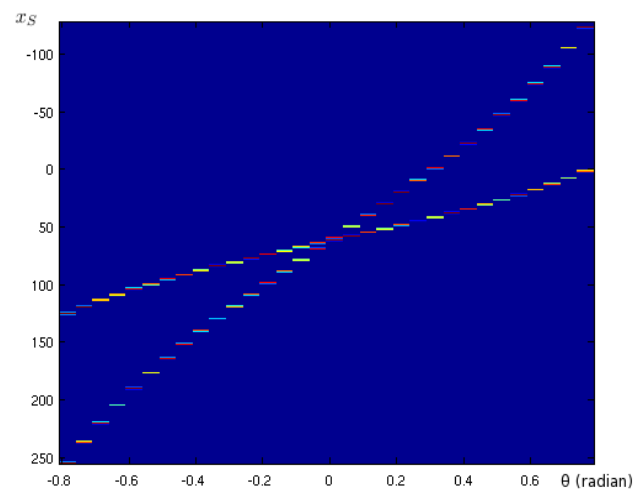

Fig. 4. The V-line Radon transform of the point object shown in Fig. 3 with $d \theta=0.005 \mathrm{rad}$. (It is also the point spread function of the transformation)

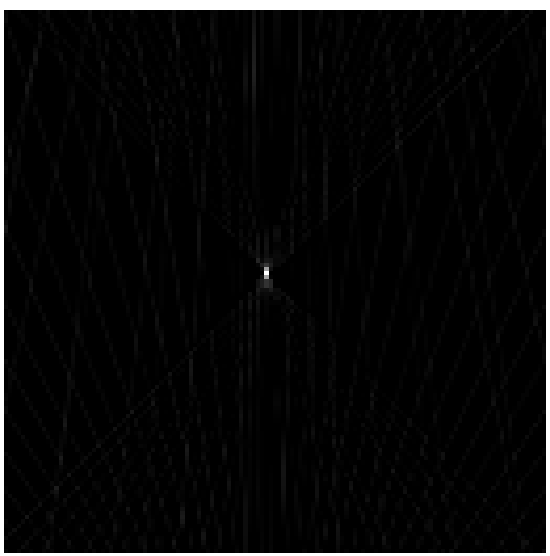

Fig. 5. Reconstruction of the point object with $d \theta=0.005 \mathrm{rad}$. In order to qualify the reconstruction quality we use the mean square error $M S E=$ $1.9 * 10^{-4}$
We note that the shape of the V-line Radon transform (or the shape of the projections) in the $\left(\theta, x_{S}\right)$-representation $\left(x_{S}\right.$ is the source position) is consistent with the arctan-curve [7]. The reconstruction of the point object is presented in Fig. 5.

The point object is well reconstructed but there are some artifacts. The use of back-projection on V-lines generates more artifacts than back-projection on straight lines in classical Radon transform. This is due to the existence of more spurious line intersections. In order to reduce these artifacts, the Hann filter $H$ is used. It is defined on the Fourier domain by its action on the first variable of a function $f$ as follows :

$$
\tilde{H} f(q, y)=\frac{|q|}{2}(1+\cos (2 \pi q)) \tilde{f}(q, y),
$$

where the Fourier transform is taken on the first variable.

Moreover for a better reconstruction, we would have to scan the angular range of reflection between 0 and $\pi / 2$. This is not possible because we would have to take away the source from the detector over huge distances. We are thus limited to a restricted angular range between 0 and $\pi / 4$ in our simulations (314 projections for every position of the source). This causes a loss of information resulting in incomplete data.

However this lack of information can be recovered by a second mirror opposite to the first one and two sets of data are used for the reconstruction (see Fig. 7, Fig. 8). We can observe an improvement on the reconstruction of the SheppLogan phantom from two sets of data (Fig. 9). There are less artifacts and the small structures in the object are clearly reconstructed.

These results illustrate undoubtedly the feasibility of the new imaging modality.

\section{CONCLUSION AND PERSPECTIVES}

In this paper, we propose a novel coupled transmissionreflection tomography based on the V-line Radon transform, which is a generalization of the classical Radon transform and can be used in biomedical imaging, in material nondestructive testing or in homeland security applications. The corresponding back-projection inversion formula generalizes the one of the ordinary Radon transform. In this new imaging the rotational motion between object and detector is replaced by the translational motion which is less stringent in some working conditions, for example for long objects or a series of object on a conveyor belt. The numerical simulation results support the feasibility and the performance of this new imaging.

The V-line Radon transform with its family of associated TV-transforms are instrumental in new possible tomographic processes involving scattering, emission and coupled transmission-reflection phenomena. More general Radon transforms on piecewise continuous curves consisting of connected pieces of arcs, e.g. arcs of circle [14], [15], as well as Radon transforms on swinging V-line around a fixed point are interesting to be considered since their inversion would open the way to new advantageous imaging processes. These topics are planned for future research. 


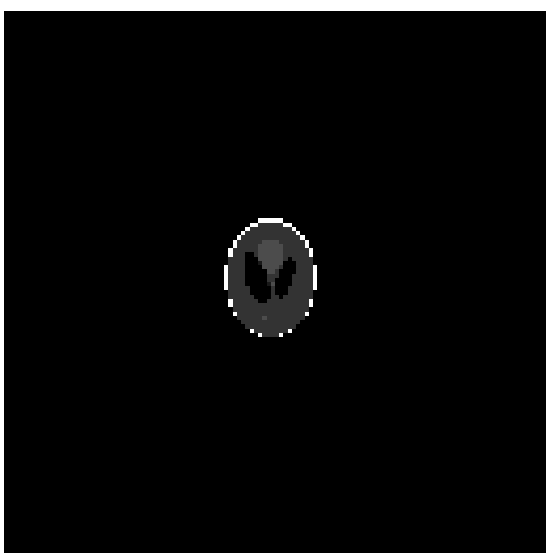

Fig. 6. Original Shepp-Logan phantom

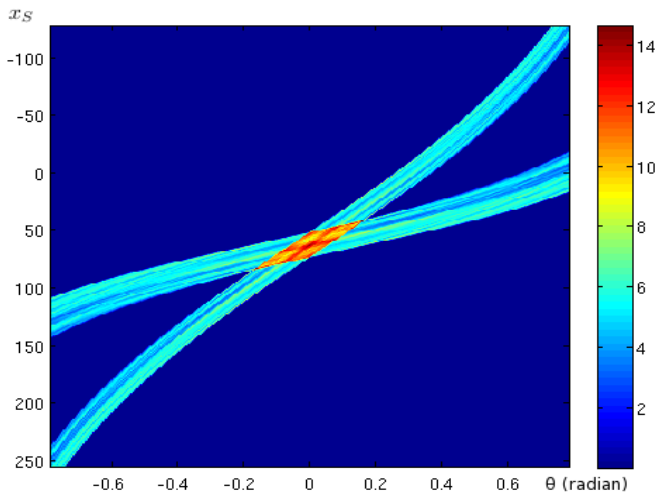

Fig. 7. The V-line Radon transform of the Shepp-Logan phantom shown in Fig. 6 with $d \theta=0.005$ rad for one set of data.

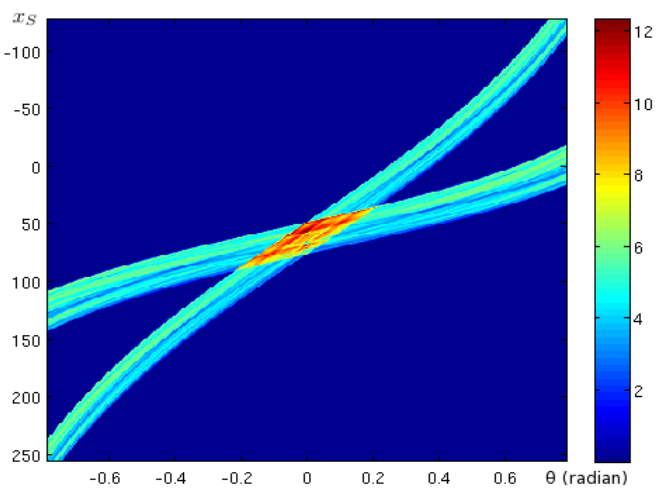

Fig. 8. The V-line Radon transform of the Shepp-Logan phantom shown in Fig. 6 with $d \theta=0.005 \mathrm{rad}$ for the second set of data.

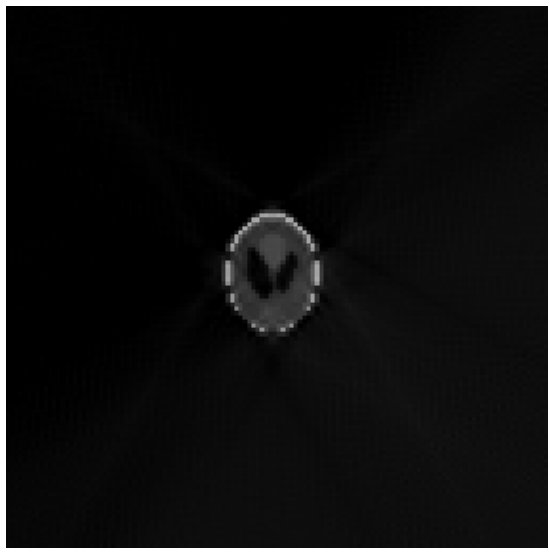

Fig. 9. Reconstruction of the Shepp-Logan phantom from the two sets of data. The $M S E=1.15 * 10^{-2}$.

\section{REFERENCES}

[1] J. Radon, “ Über die Bestimmung von Funktionnen durch ihre Integralwerte längs gewisser Mannigfaltikeiten”, Ber.Verh.Sachs.Akad.Wiss.Leipzig-Math.Natur.K1., vol. 69, p. 262 277, 1917.

[2] F. Natterer, The Mathematics of Computerized Tomography, Wiley, New York, 1986.

[3] H. H. Barrett, " The Radon Transform and its Applications ", Progress in Optics,vol. 21, p. 219-286, 1984.

[4] M. K. Nguyen and T. T. Truong ,“ Imagerie par rayonnement gamma diffus ", Hermes science Publication, 2006.

[5] M. Morvidone, M. K. Nguyen, T. T. Truong, and H. Zaidi, " On the V-line Radon transform and its imaging applications ", International Journal of Biomedical Imaging, Special issue on Mathematical methods for Images and Surfaces, 2010.

[6] M. K. Nguyen, R. Régnier, T. T. Truong and H. Zaidi, " Compton Scattering Emission Imaging Based on the $\mathrm{V}$ line Radon transform and its medical imaging applications ", IEEE Engineering in Medicine and Biology Society, 2010.

[7] R. Régnier, M. K. Nguyen and T. T. Truong, "Compton Scattering Emission Imaging Based on the V line Radon transform and its applications", the federation of European Simulation Societies, 2010.

[8] M. K Nguyen and R. Régnier, "Inversion of a new V-line Radon transform and its numerical analysis", International Conference of Numerical Analysis and Applied Mathematics 2010.

[9] R. Basko, G. L. Zeng, and G. T. Gullberg, “ Analytical reconstruction formula for the one-dimensional Compton camera ", IEEE Trans. Nucl. Sci, vol. 44, p. 1342-1346, 1997.

[10] M. K. Nguyen and T. T. Truong, " On an integral transform and its inverse in nuclear imaging ", Inverse Problems, vol. 18, p. 265-277, 2002.

[11] M. K. Nguyen, T. T. Truong, H. D. Bui, and J. L. Delarbre, " A novel inverse problem in $\gamma$-rays emission imaging ", Inverse Problems in Science and in Engineering, vol. 12, p. 225-246, 2004.

[12] T. T. Truong, M. K. Nguyen, and H. Zaidi, " The mathematical foundation of 3D Compton scatter emission imaging ", International journal of Biomedical Imaging, Special Issue on Mathematics in Biolodical Imaging, doi : 10. 1155/2007/92780, 2007.

[13] M. K. Nguyen, T. T. Truong, C. Driol, and H. Zaidi, " On a novel approach to Compton scattered emission imaging ", IEEE Transactions in Nuclear Sciences, vol. 56, p. 1430-1437, 2009.

[14] A. M. Cormack, "The Radon transform on a family of curves in the plane", Proceedings of the American Mathematical Society, vol. 83(2), p. 325-30, 1983.

[15] E. T. Quinto, "Radon transforms on curves in the plane", Lectures in Applied Mathematics: Tomography, Impedance Imaging and Integral Geometry, vol. 30, p. 231-44, 1994. 